\title{
An efficient numerical method for the solution of sliding contact problems
}

\author{
Lifeng $\mathrm{Ma}^{1,2, \ddagger}$ and Alexander M. Korsunsky ${ }^{1, *, \dagger}$ \\ ${ }^{1}$ Department of Engineering Science, Oxford University, \\ Parks Road, Oxford OX1 3PJ, U.K. \\ ${ }^{2}$ Department of Engineering Mechanics, Xi'an Jiaotong University, Xi' an 710049, China
}

\begin{abstract}
SUMMARY
In this paper, an efficient numerical method to solve sliding contact problems is proposed. Explicit formulae for the Gauss-Jacobi numerical integration scheme appropriate for the singular integral equations of the second kind with Cauchy kernels are derived. The resulting quadrature formulae for the integrals are valid at nodal points determined from the zeroes of a Jacobi polynomial. Gaussian quadratures obtained in this manner involve fixed nodal points and are exact for polynomials of degree $2 n-1$, where $n$ is the number of nodes. From this Gauss-Jacobi quadrature, the existing GaussChebyshev quadrature formulas can be easily derived. Another apparent advantage of this method is its ability to capture correctly the singular or regular behaviour of the tractions at the edge of the region of contact. Also, this analysis shows that once if the total normal load and the friction coefficient are given, the external moment $M$ and contact eccentricity $e$ (for incomplete contact) in fully sliding contact are uniquely determined. Finally, numerical solutions are computed for two typical contact cases, including sliding Hertzian contact and a sliding contact between a flat punch with rounded corners pressed against the flat surface of a semi-infinite elastic solid. These results provide a demonstration of the validity of the proposed method. Copyright (C) 2005 John Wiley \& Sons, Ltd.
\end{abstract}

KEY WORDS: singular integral equations; frictional contact problem; Gauss-Jacobi quadrature

\section{INTRODUCTION}

Contacts involving full relative sliding of mating surfaces, or gross slip, occur frequently in natural and engineering contexts. In the case known as complete contact the extent of contact is determined by the shape of the two surfaces. For contacts known as incomplete contacts, on the other hand, the extent of the contact depends on the applied tractions, both normal

*Correspondence to: Alexander M. Korsunsky, Department of Engineering Science, Oxford University, Parks Road, Oxford OX1 3PJ, U.K.

†E-mail: alexander.korsunsky@eng.ox.ac.uk

‡E-mail: malf@mail.xjtu.edu.cn 


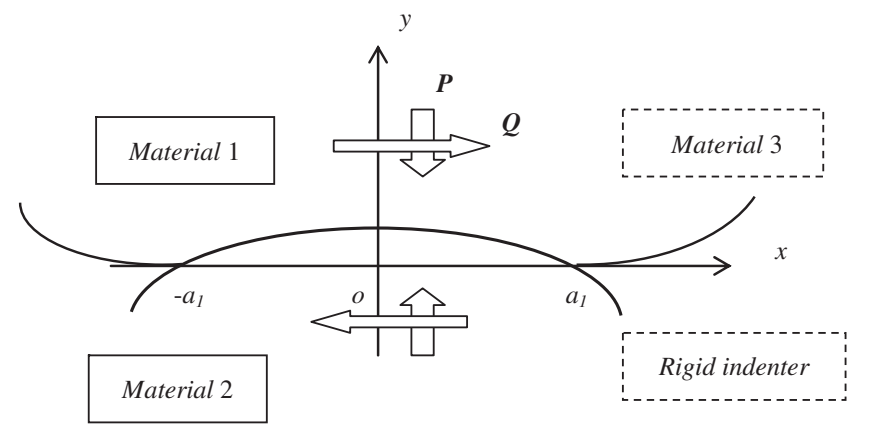

Figure 1. Example of contact geometry.

and tangential. Typical examples of plane incomplete contacts are schematically depicted in Figure 1.

The classification of contacts into complete and incomplete is the most relevant for the purposes of the present analysis, since it establishes immediate correspondence with the type of traction distribution and stress field (singular or regular) in the vicinity of the point of separation between contacting surfaces. It is also possible to introduce the term 'semi-complete contact' to refer to the two-dimensional situation when the elastic field behaviour is different at the two edges of the contact patch. At one end the contacting surfaces come together smoothly, resulting in a situation when the extent of contact is determined by the load, i.e. contact is incomplete; consequently, the stress field is regular. At the other edge of the contact patch one of the contacting profiles may exhibit a discontinuity in the derivative, resulting in a situation when, within a certain range of externally applied loads, the position of the contact edge does not change; the contact is therefore complete and the stress field singular. Note that three-dimensional contacts may display more complex behaviour than is implied by the above classification: contact may be complete along part of the edge contour of the contact patch, and incomplete along the rest; and this may change depending on the applied load.

Further possibilities for classification of contact situations exist. For example, sometimes contacts are classified into stationary, on the one hand, and receding or advancing, on the other $[1,2]$. This classification is based on the consideration of the behaviour of contact patch under increasing applied load: stationary contact remains unchanged, advancing refers to the situation when the separation point moves out with respect to the contact, and receding to the situation when the opposite takes place. This classification, however, is not entirely suitable for the present analysis, when complex loading by the combination of normal $P$ and shear $Q$ loads, and moment $M$ can be considered. The reader may readily ascertain with the help of an experiment involving e.g. a rigid wheel pressed against an elastic surface that by selecting different loading histories in terms of $P, Q, M$ it is possible to make the contact receding or advancing at will.

Let us assume that at every point within the contact full relative sliding of the contacting surfaces takes place, and that Coulomb's friction law holds in the form:

$$
q(x)=f p(x)
$$


where $q(x)$ is the shear traction, $p(x)$ is the normal traction, and $f$ is the coefficient of friction. The two contacting bodies may be elastically similar or dissimilar. In the former case the influence of shear tractions and deformations on the normal tractions can be ignored [3]. However, for dissimilar contacts that is not the case, i.e. interaction is observed between deformations induced by the normal and shear tractions. Such contact problems are said to be coupled. This type of contact can be mathematically described by the singular integral equations of the second kind $[3,4]$, which can be presented by the following standard form:

$$
a \phi(x)+\frac{b}{\pi} \int_{-1}^{1} \frac{\phi(t)}{t-x} \mathrm{~d} t=f(x), \quad x \in(-1,1)
$$

where $f(x)$ is the derivative of contact gap which will be assumed to be a continuous function, $a$ and $b$ are material parameters, and the unknown normal traction function $\phi(x)$ should be continuous and satisfy the Hölder condition. Cauchy principal value of the singular term must exist in the closed interval $[-1,1]$. For Hertzian contacts the restrictions of Equation (2) imply that the extent of contact must be small in comparison with the characteristic radii of curvature, and that the strains induced must be sufficiently small for the linear theory of elasticity to be appropriate.

For the sake of simplicity dissimilar elastic contact problems shown by materials 1 and 2 are transformed into the equivalent formulation involving a rigid-elastic contact shown by material 3 and a rigid indenter in Figure 1. The equivalent material 3 of the upper half plane in Figure 1 has the elastic properties obtained as a mathematical combination of the elastic properties of materials 1 and 2, while the indenter is assumed to be rigid [3]. The correspondence thus established is not one-to-one: a rigid-elastic contact may be represented by various dissimilar elastic contacts, but any dissimilar elastic contact can only be represented by a unique rigidelastic contact model. It should also be pointed out that the restrictions imposed in this analysis on dissimilar elastic contacts are stricter than those for the rigid-elastic contact. Here we shall use the rigid-elastic contact models to demonstrate the validity of the newly proposed numerical method.

Most contact problems involving complex geometries do not permit analytical solutions in closed form. Three principal methods have been used in such cases, namely, the finite difference method (FDM), finite element method (FEM), and boundary integral equation method (BIEM). FDM is probably the most straightforward approach involving discretization of the governing differential equations. It has been used in early studies, but is in relatively infrequent use nowadays since it is thought to provide lower precision, although some researchers argue that it is acceptable in certain situations [5]. The other two methods have been used widely to solve elastic contact problems reported in the literature. Many researchers employed general FEM packages to consider frictionless and frictional contact problems ([6-10]; and others). FEM can be effectively used for arbitrary complex geometry, but some significant effort is required to ensure that sufficiently fine mesh density is achieved near the contact face and the required accuracy of solution is achieved. BIEM has been also widely employed for this type of problems $([3,4,11]$; and others). Compared with the FEM, the BIEM is often more convenient, efficient and straightforward for simple contact configurations, but may be more restrictive in capturing complex geometry away from the contact, and complex material constitutive behaviour. Under the assumption of linear elastic deformation, the BIEM is probably the method of choice in 


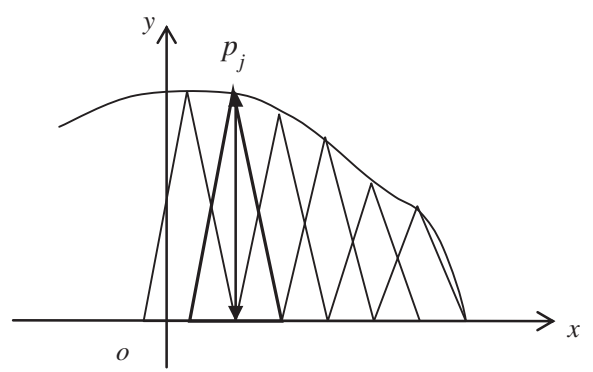

Figure 2. The triangular traction distribution method.

contact mechanics, as far as the calculation of normal and tangential traction distributions is concerned.

A numerical formation of the BIEM in contact mechanics for full slip incomplete contact problems can be constructed by taking the following steps $[3,4]$ :

1. Evaluate the derivative with respect to the tangential co-ordinate of the relative surface displacement of substrate surface due to a standard triangular distribution of normal and tangential tractions.

2. Assume a derivative with respect to the tangential co-ordinate of the vertical relative displacement (i.e. the gap function) of the indenter and substrate surface due to the combination of normal and tangential tractions.

3. Express this assumed gap function as a combination of standard triangular elements with appropriate peak values (Figure 2).

4. Use an iterative procedure involving the adjustment of the gap function values and the contact width until the required applied normal load and moment equilibrium is achieved.

The triangular element approach to discretization of the unknown continuous traction distribution is in fact equivalent to the trapezoidal rule for subinterval in numerical integration methods. In comparison with the Gaussian quadrature approach this method has somewhat lower accuracy for the same degree of discretization. Furthermore, this method does not make any explicit assumptions about the behaviour of the contact tractions near the edge of contact, despite the fact that this information is readily available from function theoretical analysis of the governing integral equation. Finally, the triangular distribution method does not provide explicit relationships between the normal load $P$, the tangential load $Q$, and the external moment $M$. A formulation of an efficient and fast numerical method similar to Gaussian quadrature approach appears to be relevant and timely.

The purpose of this paper is to analyse the singular integral equation for the case of full relative sliding elastic contact (Equation (2)), and to propose a new efficient and powerful numerical method for the solution of sliding contact problems. We also consider the closure condition that arises from the analysis, and explore the resulting relationships between the normal load $P$, the tangential load $Q$, and the externally applied moment $M$.

The authors are fully aware of the fact that the proposed method (or, more precisely, the underlying formulation) is limited to elastic problems. It is important to realize, however, 
there are many situations in engineering when contacts are designed to remain elastic. For example, the geometry of dovetails connecting aeroengine blades to the disk is optimized (by selecting cut-out angles and radii of curvature) to eliminate plastic deformation. Furthermore, elastic solutions for deformation and stress fields are widely used as an indication of the severity of contact and the likelihood of local plastic deformation, fatigue crack initiation, etc. A shining example of this approach is provided by the modern use of the linear elastic fracture mechanics: originally formulated for perfectly elastic and brittle solids, the theory has come to be applied to situations when the extent of plasticity is limited (the so-called small-scale yielding) to regions in the immediate vicinity of the fracture front. The argument is then advanced that the fracture processes occurring within this region are completely determined and governed by the deformation and stress state in the annular region surrounding the crack tip in which the material behaviour is elastic, and therefore adequately represented by the simpler linear solutions. A similar argument can be advanced in the present case: provided the extent of plasticity is very limited, the elastic formulation provides an excellent way of determining the 'outer asymptotic' description of the stress field. Demonstrating how this solution can be determined using an efficient numerical framework is the purpose of the present article.

In the present article we focus our attention on the so-called incomplete contacts defined previously. The flat-and-rounded geometry chosen in our analysis is a prominent representative case of incomplete (non-conforming) contact. However, it is important to emphasis that the method proposed is sufficiently general not only to tackle any chosen contact geometry that is incomplete, but also, with minor modifications, cases of complete contact [12-14]. This choice of flat-and-rounded geometry is neither limited nor restrictive, since it allows the consideration of a number of important cases. By modifying only one parameter (edge radius) it is possible to consider the full variety of contact geometries, from Hertzian (large edge radius) to the limiting transition towards sharp-edged, complete contact. Although in the last case the limit corresponds to a qualitative transition in the nature of the traction distribution, the approach to it can be studied using a series of flat-and-rounded geometries with diminishing edge radii. This also provides a measure of sensitivity of the solution to this parameter.

The present paper does not address partial slip contact problems (stick-slip problems). These are indeed much more challenging than the full sliding problem presently considered. However, in order to tackle these problems within the framework of coupled singular equations, it is necessary first to develop the suitable instrumentarium, particularly the quadrature techniques. The usefulness of developing well-established singular quadrature techniques for applications in fracture mechanics has been demonstrated using the example of hypersingular kernels [15]; however, similar developments have not been presented for frictional contact problems. The present paper addresses this objective.

This paper is constructed as follows. Firstly, the singular integral equation and the related integrals are introduced in Section 2. The Gauss-Jacobi numerical integration scheme for singular integral equations is derived and presented in Section 3. For the sake of completeness, the formulation appropriate to the determination of the elastic fields within the substrate is given in Section 4. In Section 5, as special cases of the result given in Section 3, the numerical formulae of Erdogan et al. [16] are easily reproduced. Finally, the efficiency of the proposed method is demonstrated using two typical contact problems in Section 6. 


\section{FUNCTIONAL FORM FOR THE SOLUTION OF SINGULAR INTEGRAL EQUATION FOR FULL RELATIVE SLIDING CONTACTS AND SOME RELATED IDENTITIES}

Muskhelishvili [17] and Erdogan et al. [16] show that the solution of Equation (2) can be expressed in terms of a weight function $w(x)$ multiplied by a bounded function $g(x)$ as follows:

$$
\phi(x)=w(x) g(x)
$$

where

$$
\begin{aligned}
w(x)= & (1-x)^{\alpha}(1+x)^{\beta} \\
\alpha= & \frac{1}{2 \pi \mathrm{i}} \ln \left(\frac{a-\mathrm{i} b}{a+\mathrm{i} b}\right)+N, \quad \beta=-\frac{1}{2 \pi \mathrm{i}} \ln \left(\frac{a-\mathrm{i} b}{a+\mathrm{i} b}\right)+M \\
& (-1<\alpha, \beta<1,-1<x<1)
\end{aligned}
$$

and $\kappa=-(\alpha+\beta)=N+M, N, M$ are integers, $\mathrm{i}=\sqrt{-1}$. In practical problems the index $\kappa$ is an integer that may assume the values $-1,0$, and 1 , depending on the nature of the problem.

We intend to represent the unknown bounded functions as a series of Jacobi polynomials in the monic form (denoted $p_{n}^{(\alpha, \beta)}(t)$ ), i.e. normalized so that the coefficient of the greatest power is equal to unity. Monic polynomials, $p_{n}^{(\alpha, \beta)}(t)$, are related to the canonical form, $P_{n}^{(\alpha, \beta)}(t)$, of the Jacobi polynomials, as follows:

$$
p_{n}^{(\alpha, \beta)}(t)=k_{n}^{-1} P_{n}^{(\alpha, \beta)}(t)
$$

where

$$
\begin{aligned}
P_{n}^{(\alpha, \beta)}(x) & =\frac{(-1)^{n}}{2^{n} n !}(1-x)^{-\alpha}(1+x)^{-\beta} \frac{\mathrm{d}^{n}}{\mathrm{~d} x^{n}}\left[(1-x)^{\alpha+n}(1+x)^{\beta+n}\right] \\
k_{n} & =\frac{\Gamma(\alpha+\beta+2 n+1)}{2^{n} n ! \Gamma(\alpha+\beta+n+1)}
\end{aligned}
$$

The following recurrence relations hold (e.g. Reference [18]):

$$
p_{n}^{(\alpha, \beta)}(x)=\left(t-b_{n}\right) p_{n-1}^{(\alpha, \beta)}(x)-c_{n} p_{n-2}^{(\alpha, \beta)}(x) \quad(n \geqslant 2)
$$

where

$$
\begin{aligned}
& b_{n}=\frac{(\alpha+\beta)(\beta-\alpha)}{(\alpha+\beta+2 n)(\alpha+\beta+2 n-2)} \\
& c_{n}=\frac{4(n-1)(\alpha+n-1)(\beta+n-1)(\alpha+\beta+n-1)}{(\alpha+\beta+2 n-1)(\alpha+\beta+2 n-2)^{2}(\alpha+\beta+2 n-3)}
\end{aligned}
$$


and

$$
p_{0}^{(\alpha, \beta)}(x)=1, \quad p_{1}^{(\alpha, \beta)}(x)=x+\frac{(\alpha-\beta)}{(\alpha+\beta+2)}
$$

Two important recurrence relations apply to the derivatives of $p_{n}^{(\alpha, \beta)}(t)$ and are given by

$$
\begin{aligned}
\left(1-x^{2}\right) p_{n}^{\prime(\alpha, \beta)}(x)= & {\left[\frac{\alpha-\beta}{\alpha+\beta+2 n}-x\right] n p_{n}^{(\alpha, \beta)}(x) } \\
& +\frac{4 n(\alpha+n)(\beta+n)(\alpha+\beta+n)}{(\alpha+\beta+2 n)^{2}(\alpha+\beta+2 n-1)} p_{n-1}^{(\alpha, \beta)}(x) \\
\frac{\mathrm{d}}{\mathrm{d} x} p_{n}^{(\alpha, \beta)}(x)= & n p_{n-1}^{(\alpha+1, \beta+1)}(x)
\end{aligned}
$$

Additionally, in the present context the following formula for Jacobi polynomials in the monic form is found useful $[12,16]$ :

$$
\int_{-1}^{1} \frac{p_{n}^{(\alpha, \beta)}(t) w(t)}{t-x} \mathrm{~d} t=\pi\left[-\frac{a}{b} w(x) p_{n}^{(\alpha, \beta)}(x)-\frac{1}{\sin \pi \alpha} p_{n-\kappa}^{(-\alpha,-\beta)}(x)\right]
$$

Equations (8), (11), (12), and (13) will be used frequently in the following sections.

\section{INTERPOLATIVE QUADRATURE FORMULAE}

We now seek the solution of Equation (2) in the form given by Equation (3). At this stage we fix the order of discretization to be an integer number $n$, and denote the roots of $p_{n}^{(\alpha, \beta)}(t)$ by $\xi_{i}(i=1,2 \ldots n)$, i.e.:

$$
p_{n}^{(\alpha, \beta)}\left(\xi_{i}\right)=0
$$

We represent the unknown function $g(t)$ in the form of a Lagrangian interpolation formula based on its values at the nodal points, $\xi_{i}$ :

$$
g(t) \approx \sum_{i=1}^{n} \frac{p_{n}^{(\alpha, \beta)}(t)}{\left(t-\xi_{i}\right) p_{n}^{\prime(\alpha, \beta)}\left(\xi_{i}\right)} g\left(\xi_{i}\right)
$$

where prime denotes the derivative.

Firstly, considering the second term on the left-hand side of Equation (2), and substituting Equation (15) gives

$$
\begin{aligned}
\int_{-1}^{1} \frac{\phi(t)}{t-x} \mathrm{~d} t & =\int_{-1}^{1} \frac{w(t) g(t)}{(t-x)} \mathrm{d} t \\
& =\int_{-1}^{1} \frac{w(t)}{(t-x)}\left[\sum_{i=1}^{n} \frac{p_{n}^{(\alpha, \beta)}(t)}{\left(t-\xi_{i}\right) p_{n}^{\prime(\alpha, \beta)}\left(\xi_{i}\right)} g\left(\xi_{i}\right)\right] \mathrm{d} t
\end{aligned}
$$




$$
\begin{aligned}
& =\sum_{i=1}^{n} \frac{g\left(\xi_{i}\right)}{p_{n}^{\prime(\alpha, \beta)}\left(\xi_{i}\right)} \int_{-1}^{1} \frac{w(t)}{(t-x)} \frac{p_{n}^{(\alpha, \beta)}(t)}{\left(t-\xi_{i}\right)} \mathrm{d} t \\
& =\sum_{i=1}^{n} \frac{g\left(\xi_{i}\right)}{p_{n}^{\prime(\alpha, \beta)}\left(\xi_{i}\right)} \frac{1}{\left(x-\xi_{i}\right)}\left\{\int_{-1}^{1} w(t) \frac{p_{n}^{(\alpha, \beta)}(t)}{(t-x)} \mathrm{d} t-\int_{-1}^{1} w(t) \frac{p_{n}^{(\alpha, \beta)}(t)}{\left(t-\xi_{i}\right)} \mathrm{d} t\right\}
\end{aligned}
$$

Equation (16) can be simplified by using Equations (13) and (14) as

$$
\int_{-1}^{1} \frac{w(t) g(t)}{(t-x)} \mathrm{d} t=\sum_{i=1}^{n} \frac{g\left(\xi_{i}\right)}{p_{n}^{\prime(\alpha, \beta)}\left(\xi_{i}\right)} \frac{\pi}{\left(x-\xi_{i}\right)}\left[\begin{array}{l}
-\frac{a}{b} w(x) p_{n}^{(\alpha, \beta)}(x)-\frac{1}{\sin \pi \alpha} p_{n-\kappa}^{(-\alpha,-\beta)}(x) \\
+\frac{1}{\sin \pi \alpha} p_{n-\kappa}^{(-\alpha,-\beta)}\left(\xi_{i}\right)
\end{array}\right]
$$

or

$$
\begin{aligned}
\int_{-1}^{1} \frac{w(t) g(t)}{(t-x)} \mathrm{d} t= & -\pi \frac{a}{b} w(x) \sum_{i=1}^{n} \frac{g\left(\xi_{i}\right)}{p_{n}^{\prime(\alpha, \beta)}\left(\xi_{i}\right)} \frac{p_{n}^{(\alpha, \beta)}(x)}{\left(x-\xi_{i}\right)} \\
& +\sum_{i=1}^{n} \frac{g\left(\xi_{i}\right)}{p_{n}^{\prime(\alpha, \beta)}\left(\xi_{i}\right)} \frac{\pi}{\left(x-\xi_{i}\right)} \frac{\left[p_{n-\kappa}^{(-\alpha,-\beta)}\left(\xi_{i}\right)-p_{n-\kappa}^{(-\alpha,-\beta)}(x)\right]}{\sin \pi \alpha}
\end{aligned}
$$

By virtue of Equation (15), we can write Equation (18) as

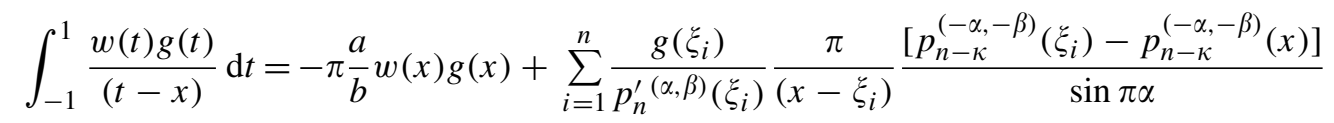

Substituting Equation (19) into Equation (2), and considering Equation (3), the following discretized form of Equation (2) can be derived:

$$
\sum_{i=1}^{n} \frac{g\left(\xi_{i}\right)}{p_{n}^{\prime(\alpha, \beta)}\left(\xi_{i}\right)} \frac{b}{\left(x-\xi_{i}\right)} \frac{1}{\sin \pi \alpha}\left[p_{n-\kappa}^{(-\alpha,-\beta)}\left(\xi_{i}\right)-p_{n-\kappa}^{(-\alpha,-\beta)}(x)\right]=f(x)
$$

We have replaced the original equation Equation (2) by an approximate form containing only the values of $g(t)$ at $n$ fixed collocation points $\xi_{i}$.

We now wish to introduce a further approximation, and solve Equation (20) by enforcing the equality only at a finite number of collocation points $x=x_{j}$. We require a judicious choice of collocation points to achieve an efficient solution. By choosing in Equation (20) $x=x_{j}$, where $x_{j}$ are

$$
p_{n-\kappa}^{(-\alpha,-\beta)}\left(x_{j}\right)=0 \quad(j=1,2,3, \ldots, n-\kappa)
$$

Equation (2) is finally reduced to the following algebraic system:

$$
\sum_{i=1}^{n} \frac{W\left(\xi_{i}\right)}{\sin \pi \alpha} \frac{b}{\left(x_{j}-\xi_{i}\right)} g\left(\xi_{i}\right)=f\left(x_{j}\right)
$$


where

$$
W\left(\xi_{i}\right)=\frac{p_{n-\kappa}^{(-\alpha,-\beta)}\left(\xi_{i}\right)}{p_{n}^{\prime(\alpha, \beta)}\left(\xi_{i}\right)}
$$

Here the $n$ nodal points $\xi_{i}(i=1,2,3 \ldots n)$ satisfy $p_{n}^{(\alpha, \beta)}\left(\xi_{i}\right)=0$, while the $(n-\kappa)$ collocation points $x_{j}(j=1,2,3, \ldots, n-\kappa)$ satisfy $p_{n-\kappa}^{(-\alpha,-\beta)}\left(x_{j}\right)=0$.

Equation (22) is the Gauss-Jacobi quadrature formula for singular integral equations applicable to the general type of behaviour of the solution, including the cases when the value of the unknown function $\phi(x)=w(x) g(x)$ are finite or approach infinity at either or both ends of the interval.

Contact problems can be classified into three kinds according to the value of $\kappa$ as follows:

(1) Complete contact $(\kappa=1)$.

In this case the approximate formulation of Equation (22) is not closed, since the number of equation is less than the number of unknowns by one. An additional condition should be considered. For complete contacts, the equilibrium condition can be viewed as the additional condition as follows:

$$
P=a_{1} \int_{-1}^{1} \phi(t) \mathrm{d} t
$$

where $P$ is the external force, and $a_{1}$ is the half-contact width.

Making use of Equation (15) and considering Equations (13) and (14), Equation (24) is transformed into the form

$$
\sum_{i=1}^{n} W\left(\xi_{i}\right) g\left(\xi_{i}\right)=-\frac{\sin \pi \alpha}{\pi} \frac{P}{a_{1}}
$$

From Equations (22) and (25), the traction along the contact surface can be solved.

(2) Semi-complete contact $(\kappa=0)$.

The number of unknown variables are equal to the number of equations in Equation (24), so the system of equations is closed. This case can be easily solved, and a simple example has been investigated by Erdogan et al. [16].

(3) Incomplete contact $(\kappa=-1)$.

Equation (22) is satisfied for $n+1$ values of $x_{j}$. Since there are only $n$ unknowns $g\left(\xi_{i}\right)$ in (22), an equation corresponding to one particular value of $x_{j}$ must be ignored. More importantly, it should be emphasized that for incomplete contacts not only the extent of contact, but also its centre position are not known. It follows that the gap function must in fact be taken for shifted form $f(x)=f_{1}(x-e)$, where $e$ denotes the contact's eccentricity. For this case, Muskhelishvili [17] shows that an additional condition must be satisfied,

$$
\int_{-1}^{1} \frac{f_{1}(x-e)}{w(x)} \mathrm{d} x=0
$$

From Equation (26) the eccentricity $e$ can be computed.

Once the contact type and gap function $f(x)$ are known, other unknown variables, such as surface tractions and the substrate stress state, can be uniquely determined. The external 
moment acting on the contact surface can be determined as

$$
M=a_{1}^{2} \int_{-1}^{1} t \phi(t) \mathrm{d} t
$$

This observation implies that once if the total normal load and the friction coefficient are given, the external moment $M$ in a fully sliding contact is uniquely determined by the solution. Substituting Equations (3) and (15) into Equation (27), and considering the orthogonality property of Jacobi polynomials, and Equation (14), Equation (27) can be numerically expressed as follows:

$$
\begin{aligned}
M & =a_{1}^{2} \int_{-1}^{1} t \phi(t) \mathrm{d} t=a_{1}^{2} \int_{-1}^{1} t w(t) g(t) \mathrm{d} t=a_{1}^{2} \int_{-1}^{1}\left[\sum_{i=1}^{n} \frac{t w(t) p_{n}^{(\alpha, \beta)}(t)}{\left(t-\xi_{i}\right) p_{n}^{\prime(\alpha, \beta)}\left(\xi_{i}\right)} g\left(\xi_{i}\right)\right] \mathrm{d} t \\
& =a_{1}^{2} \sum_{i=1}^{n} \frac{g\left(\xi_{i}\right)}{p_{n}^{\prime(\alpha, \beta)}\left(\xi_{i}\right)} \int_{-1}^{1} \frac{t w(t) p_{n}^{(\alpha, \beta)}(t)}{\left(t-\xi_{i}\right)} \mathrm{d} t \\
& =a_{1}^{2} \sum_{i=1}^{n} \frac{g\left(\xi_{i}\right)}{p_{n}^{\prime(\alpha, \beta)}\left(\xi_{i}\right)}\left\{\int_{-1}^{1} w(t) p_{n}^{(\alpha, \beta)}(t) \mathrm{d} t+\xi_{i} \int_{-1}^{1} \frac{w(t) p_{n}^{(\alpha, \beta)}(t)}{\left(t-\xi_{i}\right)} \mathrm{d} t\right\} \\
& =-\frac{\pi a_{1}^{2}}{\sin \pi \alpha} \sum_{i=1}^{n} \xi_{i} \frac{p_{n-\kappa}^{(-\alpha,-\beta)}\left(\xi_{i}\right) g\left(\xi_{i}\right)}{p_{n}^{\prime(\alpha, \beta)}\left(\xi_{i}\right)} \\
& =-\frac{\pi a_{1}^{2}}{\sin \pi \alpha} \sum_{i=1}^{n} \xi_{i} W\left(\xi_{i}\right) g\left(\xi_{i}\right)
\end{aligned}
$$

The problem of finding polynomial roots to a great degree of accuracy is of direct relevance to the task of accurate determination of the Gaussian weights [19] and hence the precision of the resulting quadrature. Efficient numerical algorithms are available for recursive evaluation of Jacobi polynomials and for finding their roots with a high degree of precision by iterative improvement. These algorithms are given by Stroud and Secrest [18].

\section{STRESS FIELD IN THE SUBSTRATE}

For the sake of completeness of presentation, the procedure for the calculation of the stress field within the substrate is presented here.

Suppose the solution has been found for the surface tangential traction $q(\xi)$ and normal traction $p(\xi)$ within the contact by the previous method. The stress fields in the substrate can be obtained by the Green function method as follows:

$$
\sigma_{i j}(x, y)=\int_{-1}^{1} p(\xi) k_{i j y}(\xi, x, y) \mathrm{d} \xi+\int_{-1}^{1} q(\xi) k_{i j x}(\xi, x, y) \mathrm{d} \xi \quad(i, j=1,2)
$$


The influence functions are regular for all points not lying on the boundary and are given by [3]

$$
\begin{array}{ll}
k_{11 y}(\xi, x, y)=\frac{2}{\pi} \frac{(x-\xi)^{2} y}{\left[(x-\xi)^{2}+y^{2}\right]^{2}}, & k_{11 x}(\xi, x, y)=\frac{2}{\pi} \frac{(x-\xi)^{3}}{\left[(x-\xi)^{2}+y^{2}\right]^{2}} \\
k_{22 y}(\xi, x, y)=\frac{2}{\pi} \frac{y^{3}}{\left[(x-\xi)^{2}+y^{2}\right]^{2}}, & k_{22 x}(\xi, x, y)=\frac{2}{\pi} \frac{(x-\xi) y^{2}}{\left[(x-\xi)^{2}+y^{2}\right]^{2}} \\
k_{12 y}(\xi, x, y)=\frac{2}{\pi} \frac{(x-\xi) y^{2}}{\left[(x-\xi)^{2}+y^{2}\right]^{2}}, & k_{12 x}(\xi, x, y)=\frac{2}{\pi} \frac{(x-\xi)^{2} y}{\left[(x-\xi)^{2}+y^{2}\right]^{2}}
\end{array}
$$

By virtue of Equation (1) and using the fact that within the contact region $p(x)=\phi(x)$, Equation (29) can also be rewritten as

$$
\sigma_{i j}(x, y)=\int_{-1}^{1} \phi(\xi)\left[k_{i j y}(\xi, x, y)+f k_{i j x}(\xi, x, y)\right] \mathrm{d} \xi
$$

Substituting Equations (3), (15) into Equation (31), and using Equations (13) and (14), we obtain

$$
\begin{aligned}
\sigma_{i j}(x, y) & =\int_{-1}^{1} \phi(\xi)\left[k_{i j y}(\xi, x, y)+f k_{i j x}(\xi, x, y)\right] \mathrm{d} \xi \\
& =-\frac{\pi}{\sin \pi \alpha} \sum_{m=1}^{n} \frac{p_{n-\kappa}^{(-\alpha,-\beta)}\left(\xi_{m}\right)}{p_{n}^{\prime(\alpha, \beta)}\left(\xi_{m}\right)}\left[k_{i j y}\left(\xi_{m}, x, y\right)+f k_{i j x}\left(\xi_{m}, x, y\right)\right] g\left(\xi_{m}\right) \\
& =-\frac{\pi}{\sin \pi \alpha} \sum_{m=1}^{n} W\left(\xi_{m}\right)\left[k_{i j y}\left(\xi_{m}, x, y\right)+f k_{i j x}\left(\xi_{m}, x, y\right)\right] g\left(\xi_{m}\right)
\end{aligned}
$$

\section{SPECIAL CASES OF THE GAUSS-JACOBI QUADRATURE}

The general Gauss-Jacobi quadrature formulae presented in Section 3 contain as special cases some of the published results, e.g. the formulae for singular integral equations of the first kind by Erdogan et al. [16] suitable for the analysis of frictionless contacts. We can elucidate the nature of Gauss-Jacobi quadrature formulae somewhat further in this connection.

The standard form of singular integral equation of the first kind is obtained by setting $a=0, b=1$. Different cases for the values of $\alpha, \beta$, and $\kappa$ can be obtained from Equation (4) corresponding to the contact type as described in Section 3:

1. For $\alpha=\beta=-\frac{1}{2}, \kappa=-(\alpha+\beta)=1$. 
The expression for the Gaussian quadrature weights in Equation (23) is simplified to

$$
W\left(\xi_{i}\right)=\frac{p_{n-1}^{(-\alpha,-\beta)}\left(\xi_{i}\right)}{p_{n}^{\prime(\alpha, \beta)}\left(\xi_{i}\right)}=\frac{p_{n-1}^{(-\alpha,-\beta)}\left(\xi_{i}\right)}{n p_{n-1}^{(1+\alpha, 1+\beta)}\left(\xi_{i}\right)}=\frac{p_{n-1}^{(1 / 2,1 / 2)}\left(\xi_{i}\right)}{n p_{n-1}^{(1 / 2,1 / 2)}\left(\xi_{i}\right)}=\frac{1}{n}
$$

where

$$
p_{n}^{(1 / 2,1 / 2)}(x)=\frac{2^{n+1} n ! \Gamma\left(n+\frac{3}{2}\right)}{\Gamma(2 n+2) \sqrt{\pi}} U_{n}(x)
$$

i.e. the monic form of the Chebyshev polynomial of the second type, was used. Hence, Equations (22) and (25), as well as Equations (14) and (21) can, respectively, be reduced to the following forms:

$$
\begin{aligned}
\sum_{i=1}^{n} \frac{1}{n} \frac{1}{\left(\xi_{i}-x_{j}\right)} g\left(\xi_{i}\right) & =f\left(x_{j}\right) \\
\sum_{i=1}^{n} \frac{\pi}{n} g\left(\xi_{i}\right) & =\frac{P}{a_{1}}
\end{aligned}
$$

where

$$
\begin{aligned}
& T_{n}\left(\xi_{i}\right)=0, \quad \xi_{i}=\cos \left(\frac{2 i-1}{2 n} \pi\right) \quad(i=1, \ldots, n) \\
& U_{n-1}\left(x_{j}\right)=0, \quad x_{j}=\cos \frac{\pi j}{n} \quad(j=1, \ldots, n-1)
\end{aligned}
$$

and the relation

$$
p_{n}^{(-1 / 2,-1 / 2)}(x)=\frac{2^{n} \Gamma(n) \Gamma\left(n+\frac{1}{2}\right)}{\Gamma(2 n) \sqrt{\pi}} T_{n}(x)
$$

is used for the monic form of the Chebyshev polynomial of the first kind.

2. For $\alpha=\beta=\frac{1}{2}, \kappa=-(\alpha+\beta)=-1$.

Using Equations (34), (39) and the following relation:

$$
\frac{\mathrm{d}}{\mathrm{d} x} p_{n}^{(1 / 2,1 / 2)}(\xi)=\frac{2^{n+1} n ! \Gamma\left(n+\frac{3}{2}\right)}{\Gamma(2 n+2) \sqrt{\pi}} U_{n}^{\prime}(\xi)=\frac{2^{n+1} n ! \Gamma\left(n+\frac{3}{2}\right)}{\Gamma(2 n+2) \sqrt{\pi}}\left[\frac{x U_{n}(\xi)-(n+1) T_{n+1}(\xi)}{1-\xi^{2}}\right]
$$

as well as

$$
p_{n}^{(1 / 2,1 / 2)}\left(\xi_{i}\right)=\frac{2^{n+1} n ! \Gamma\left(n+\frac{3}{2}\right)}{\Gamma(2 n+2) \sqrt{\pi}} U_{n}\left(\xi_{i}\right)=0
$$

the Gaussian quadrature weights, Equation (23) can be simplified as

$$
W\left(\xi_{i}\right)=\frac{p_{n-\kappa}^{(-\alpha,-\beta)}\left(\xi_{i}\right)}{p_{n}^{\prime(\alpha, \beta)}\left(\xi_{i}\right)}=\frac{p_{n+1}^{(-1 / 2,-1 / 2)}\left(\xi_{i}\right)}{p_{n}^{\prime(1 / 2,1 / 2)}\left(\xi_{i}\right)}=\frac{\left(\xi_{i}^{2}-1\right)}{(n+1)}
$$


Hence, Equations (22), (14) and (21) can, respectively, be transformed into

$$
\begin{gathered}
\sum_{i=1}^{n} \frac{\left(1-\xi_{i}^{2}\right)}{(n+1)} \frac{1}{\left(\xi_{i}-x_{j}\right)} g\left(\xi_{i}\right)=f\left(x_{j}\right) \\
U_{n}\left(\xi_{i}\right)=0, \quad \xi_{i}=\cos \left(\frac{i \pi}{n+1}\right) \quad(i=1, \ldots, n) \\
T_{n+1}\left(x_{j}\right)=0, \quad x_{j}=\cos \left(\frac{\pi}{2} \frac{2 j-1}{n+1}\right) \quad(j=1, \ldots, n+1)
\end{gathered}
$$

3. For $\alpha=\frac{1}{2}, \beta=-\frac{1}{2}, \kappa=-(\alpha+\beta)=0$.

$$
\begin{aligned}
& p_{n}^{(1 / 2,-1 / 2)}(x)=\frac{2^{n} n ! \Gamma(n+1)}{\Gamma(2 n+1)} \cdot \frac{1 \cdot 3 \cdot 5 \cdots(2 n-1)}{2 \cdot 4 \cdot 6 \cdots(2 n)} \frac{\sin (((2 n+1) / 2) \theta)}{\sin \left(\frac{1}{2} \theta\right)}, \quad x=\cos \theta \\
& p_{n}^{(-1 / 2,1 / 2)}(x)=\frac{2^{n} n ! \Gamma(n+1)}{\Gamma(2 n+1)} \cdot \frac{1 \cdot 3 \cdot 5 \cdots(2 n-1)}{2 \cdot 4 \cdot 6 \cdots(2 n)} \frac{\cos (((2 n+1) / 2) \theta)}{\cos \left(\frac{1}{2} \theta\right)}, \quad x=\cos \theta
\end{aligned}
$$

Using Equations (11), (14), (45) and (46), the quadrature weight formula is obtained as

$$
W\left(\xi_{i}\right)=\frac{p_{n-\kappa}^{(-\alpha,-\beta)}\left(\xi_{i}\right)}{p_{n}^{\prime(\alpha, \beta)}\left(\xi_{i}\right)}=\frac{p_{n}^{(-1 / 2,1 / 2)}\left(\xi_{i}\right)}{p_{n}^{\prime(1 / 2,-1 / 2)}\left(\xi_{i}\right)}=\frac{4\left(1-\xi_{i}^{2}\right) p_{n}^{(-1 / 2,1 / 2)}\left(\xi_{i}\right)}{(2 n+1) p_{n-1}^{(1 / 2,-1 / 2)}\left(\xi_{i}\right)}=-\frac{2\left(1-\xi_{i}\right)}{(2 n+1)}
$$

Substituting Equation (47) into Equation (22) gives

$$
\sum_{i=1}^{n} \frac{2\left(1-\xi_{i}\right)}{(2 n+1)} \frac{1}{\left(\xi_{i}-x_{j}\right)} g\left(\xi_{i}\right)=f\left(x_{j}\right)
$$

where

$$
\begin{aligned}
& p_{n}^{(-1 / 2,1 / 2)}\left(x_{j}\right)=0, \quad x_{j}=\cos \left(\frac{2 j-1}{2 n+1} \pi\right), \quad j=1,2,3 \ldots n \\
& p_{n}^{(1 / 2,-1 / 2)}\left(\xi_{i}\right)=0, \quad \xi_{i}=\cos \left(\frac{2 \pi \mathrm{i}}{2 n+1}\right), \quad i=1,2,3 \ldots n
\end{aligned}
$$

4. For $\alpha=-\frac{1}{2}, \beta=\frac{1}{2}, \kappa=-(\alpha+\beta)=0$.

The Gauss-Jacobi quadrature formulae for this case are obtained as

$$
\begin{aligned}
W\left(\xi_{i}\right)= & \frac{p_{n-\kappa}^{(-\alpha,-\beta)}\left(\xi_{i}\right)}{p_{n}^{\prime(\alpha, \beta)}\left(\xi_{i}\right)}=\frac{p_{n}^{(1 / 2,-1 / 2)}\left(\xi_{i}\right)}{p_{n}^{\prime(-1 / 2,1 / 2)}\left(\xi_{i}\right)}=\frac{2\left(1+\xi_{i}\right)}{(2 n+1)} \\
& \sum_{i=1}^{n} \frac{2\left(1+\xi_{i}\right)}{(2 n+1)} \frac{1}{\left(\xi_{i}-x_{j}\right)} g\left(\xi_{i}\right)=f\left(x_{j}\right)
\end{aligned}
$$


where

$$
\begin{aligned}
& p_{n}^{(1 / 2,-1 / 2)}\left(x_{j}\right)=0, \quad x_{j}=\cos \left(\frac{2 \pi j}{2 n+1}\right), \quad j=1,2,3 \ldots n \\
& p_{n}^{(-1 / 2,1 / 2)}\left(\xi_{i}\right)=0, \quad \xi_{i}=\cos \left(\frac{2 i-1}{2 n+1} \pi\right), \quad i=1,2,3 \ldots n
\end{aligned}
$$

Some linear algebraic equations are identical in form to those given by Erdogen et al. [16]. However, the derivation on the basis of the general results presented in Section 3 is much simplified. The powerful quadrature formulae for the equations of the first kind have been used extensively in the solution of fracture mechanics and frictionless contact mechanics problems. We express the expectation that the Gauss-Jacobi quadrature formulae for singular integral equations of the second kind, proposed in this paper, will serve as a convenient analysis tool in these fields.

\section{EXAMPLE PROBLEMS}

The special cases discussed in Section 5 serve as an illustration of the correctness of the proposed Gauss-Jacobi quadrature formulae in special cases. In this section two further numerical examples drawn from typical sliding contact problems will be considered. Firstly the sliding Hertzian contact in the presence of friction is solved. Secondly, fully sliding contact is considered between a rigid flat punch with rounded corner and an elastic substrate. Frictionless contact for this case has been investigated by Ciavarella et al. [20].

\subsection{Sliding Hertzian contact with friction force}

The problem of a rigid cylindrical punch sliding on an elastic half-plane is considered in Figure 3. The problem is solved by the numerical method presently proposed. Note, however, that this problem may also be solved in closed form analytically. Comparing the results obtained

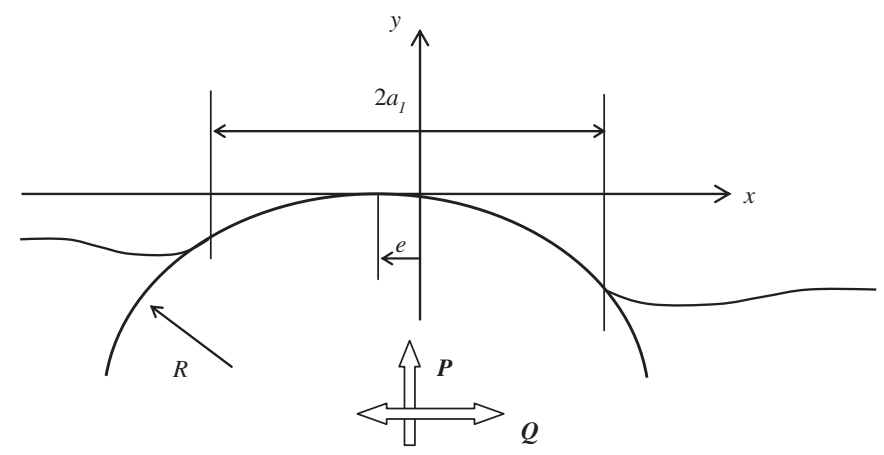

Figure 3. Frictional Hertzian contact. 
by the two different approaches provides a good method for the evaluation of the accuracy of the numerical method proposed in this paper.

Since the punch is rigid we are concerned solely with the deformation of the half-plane. The normal displacement of the surface, $v(x)$, is related to the normal and shear tractions $p(x)$ and $q(x)$ as follows [21]

$$
-\frac{1-2 v}{2 \mu} q(x)+\frac{1-v}{\pi \mu} \int_{-a_{1}}^{a_{1}} \frac{p(t)}{t-x} \mathrm{~d} t=\frac{\partial v(x)}{\partial x}=-\frac{(x-e)}{R}
$$

where $v$ is Poisson's ratio, $\mu$ is the modulus of rigidity, $R$ is the radius of the contacting cylinder and $e$ is the eccentricity. Since the punch is sliding, the shear and normal tractions are everywhere related by Equation (1). Hence, Equation (53) becomes

$$
-f \frac{1-2 v}{2(1-v)} p(x)+\frac{1}{\pi} \int_{-a_{1}}^{a_{1}} \frac{p(t)}{t-x} \mathrm{~d} t=-\frac{\mu}{(1-v)} \frac{(x-e)}{R}
$$

The eccentricity $e$ in Equation (54) can be found from the following auxiliary equation [21]:

$$
\int_{-a_{1}}^{a_{1}} \frac{(x-e)}{\left(a_{1}-x\right)^{B}\left(a_{1}+x\right)^{1-B}} \mathrm{~d} x=0
$$

as

$$
e=a_{1}(2 B-1)
$$

The solution of Equation (54) is [21]

$$
\begin{aligned}
p(x) & =\frac{\mu a_{1}}{(1-v) R} \sin B \pi\left(1-\frac{x}{a_{1}}\right)^{B}\left(1+\frac{x}{a_{1}}\right)^{1-B} \\
& =\frac{\mu a_{1}}{(1-v) R} \sin B \pi(1-X)^{B}(1+X)^{1-B} \\
& =\phi(X)
\end{aligned}
$$

where, $X=x / a_{1}, B$ may be determined by

$$
\tan \pi B=-\frac{2(1-v)}{f(1-2 v)} \quad(0<B<1)
$$

and

$$
a_{1}=\sqrt{\frac{P R}{2 B(1-B)} \frac{(1-v)}{\pi \mu}}
$$

where $P$ is the external load. 
Table I. Collocation points and numerical results for $g\left(\xi_{i}\right)$.

\begin{tabular}{rcc}
\hline$i$ & $\xi_{i}$ & $g\left(\xi_{i}\right)$ \\
\hline 1 & $0.98910345 E+00$ & $0.11549526 E+08$ \\
2 & $0.95614121 E+00$ & $0.11549529 E+08$ \\
3 & $0.90182038 E+00$ & $0.11549531 E+08$ \\
4 & $0.82735382 E+00$ & $0.11549532 E+08$ \\
5 & $0.73440489 E+00$ & $0.11549531 E+08$ \\
6 & $0.62504990 E+00$ & $0.11549532 E+08$ \\
7 & $0.50173164 E+00$ & $0.11549533 E+08$ \\
8 & $0.36720485 E+00$ & $0.11549533 E+08$ \\
9 & $0.22447462 E+00$ & $0.11549533 E+08$ \\
10 & $0.76729309 E-01$ & $0.11549531 E+08$ \\
11 & $-0.72730694 E-01$ & $0.11549532 E+08$ \\
12 & $-0.22056670 E+00$ & $0.11549533 E+08$ \\
13 & $-0.36347630 E+00$ & $0.11549532 E+08$ \\
14 & $-0.49826712 E+00$ & $0.11549531 E+08$ \\
15 & $-0.62192817 E+00$ & $0.11549531 E+08$ \\
16 & $-0.73169705 E+00$ & $0.11549532 E+08$ \\
17 & $-0.82512169 E+00$ & $0.11549531 E+08$ \\
18 & $-0.90011513 E+00$ & $0.11549531 E+08$ \\
19 & $-0.95500205 E+00$ & $0.11549533 E+08$ \\
20 & $-0.98855580 E+00$ & $0.11549521 E+08$ \\
\hline
\end{tabular}

From Equations (3), (4) and (57), the explicit solution for the bounded function $g(x)$ is found:

$$
g(X)=\frac{\mu a_{1}}{(1-v) R} \sin B \pi
$$

which for this case is a constant.

Equation (54) can also be written in the standard form as

$$
-f \frac{1-2 v}{2(1-v)} \phi(X)+\frac{1}{\pi} \int_{-1}^{1} \frac{\phi(T)}{T-X} \mathrm{~d} T=-\frac{\mu}{(1-v)} \frac{\left(a_{1} X-e\right)}{R}
$$

The numerical solution of Equation (61), i.e. the nodal values $g\left(T_{i}\right)$, obtained by the GaussJacobi quadrature formulae, Equation (25), is presented in Table I. The calculations were performed for $f=-0.3, v=0.3, E=2 \mu(1+v)=1.15 \times 10^{11} \mathrm{~Pa}, P=1000 \mathrm{~N} / \mathrm{m}, R=0.3 \mathrm{~m}$, the number of collocation points was $n=20$, and the nodal points $\xi_{i}$ were calculated from Equation (14). From Equation (58) and the specified parameters, $B$ has the value $B=0.4727828$, so that the analytical solution gives

$$
g(X)=0.11549532 \times 10^{8}
$$

Under the chosen total load $P$, using the Newton iteration method, we evaluate the semi-contact width and the external moment, respectively, as $a_{1}=55.036 \mu \mathrm{m}$ and $M=0 \mathrm{~N} \mathrm{~m}$.

The calculated nodal values of the function $g\left(\xi_{i}\right)$ given in Table I compare well with the analytical result in Equation (62). Numerical results in Table I provide a clear demonstration that the proposed method is an efficient technique for the solution of singular integral equations of the second kind frequently encountered in contact mechanics. 


\subsection{Fully sliding contact of a flat punch with rounded corners}

The problem of a fully sliding contact between the flat semi-infinite elastic substrate and a rigid flat punch with rounded corners, shown in Figure 4, is described by the equation

$$
-f \frac{1-2 v}{2(1-v)} \phi(x)+\frac{1}{\pi} \int_{-a_{1}}^{a_{1}} \frac{\phi(t)}{t-x} \mathrm{~d} t=-\frac{\mu}{(1-v)} f(x)
$$

where the derivative of gap function $f(x)$ of the punch can be expressed as

$$
f(x)= \begin{cases}-\frac{x+r+e}{R}, & x \in\left[-a_{1},-(r+e)\right] \\ 0, & x \in[-(r+e),(r-e)] \\ -\frac{x-(r-e)}{R}, & x \in\left[(r-e), a_{1}\right]\end{cases}
$$

where $r$ is the half-width of the flat end. The eccentricity $e$ requires numerical determination from Equation (26). We carried out calculations for $v=0.33, E=2 \mu(1+v)=1.15 \times 10^{11} \mathrm{~Pa}$, $R=50 \mathrm{~mm}, r=4.15 \mathrm{~mm}, P=1.5 \times 10^{6} \mathrm{~N} / \mathrm{m}$.

Figure 5 shows the traction profiles along the contact. Detailed numerical results for the eccentricity, extent of contact, and external moment are given in Table II. With increasing coefficient of friction the eccentricity increases, as does the external moment. At the same time, the value of peak traction on the left (trailing edge of contact) decreases while the value of peak traction on the right (leading edge of contact) increases. Compared with the value of peak traction in the frictionless case, the peak traction value increases by about $20 \%$ for the friction coefficient of $f=-0.8$. In many engineering problems the absolute value of the friction coefficient is not expected to exceed 0.6. From this numerical result we conclude that the effect of friction on the normal traction distribution may be judged to be mild for those cases. This assumption has been frequently used in friction contact mechanics without direct proof. However, if the friction coefficient increases significantly, as in cases of severe and scoffing,

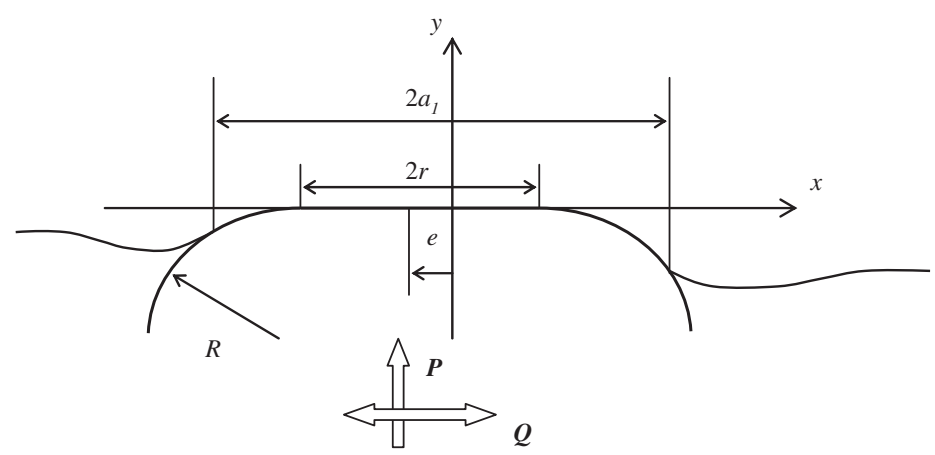

Figure 4. Contact of rigid flat punch with rounded corners and the punch geometry. 


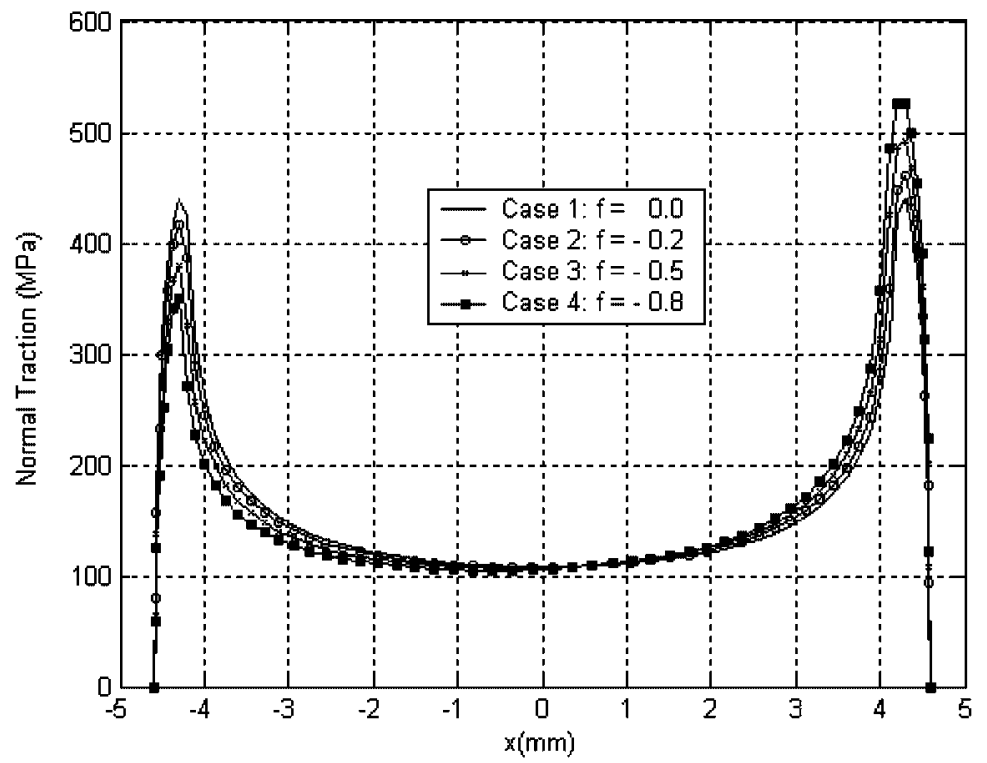

Figure 5. Normal traction distributions for a rigid flat punch with rounded corners sliding over a half-plane under normal load $P=1.5 \mathrm{MN} / \mathrm{m}$, shown for different values of the friction coefficient.

Table II. Variation of contact parameters with the friction coefficient for a rigid flat punch with rounded corners sliding over a half-plane under normal load $P=1.5 \mathrm{MN} / \mathrm{m}$.

\begin{tabular}{lccc}
\hline $\begin{array}{l}\text { Friction } \\
\text { coefficient, } f\end{array}$ & $\begin{array}{c}\text { Contact half-width, } \\
a_{1}(\mathrm{~mm})\end{array}$ & $\begin{array}{c}\text { Eccentricity } \\
\text { parameter, } e(\mu \mathrm{m})\end{array}$ & $\begin{array}{c}\text { External moment, } \\
M(\mathrm{kN} \mathrm{m})\end{array}$ \\
\hline Case 1: $f=0.0$ & 4.590 & 0.0 & 0.0 \\
Case 2: $f=-0.2$ & 4.591 & 26.8 & 0.1997 \\
Case 3: $f=-0.5$ & 4.595 & 67.0 & 0.4972 \\
Case 4: $f=-0.8$ & 4.601 & 106.8 & 0.7882 \\
\hline
\end{tabular}

wear the tangential tractions take on substantial values, and the associated external moment is also increased. The combination of tangential and normal tractions induces strong effects on the substrate stress state, resulting in situations when critical stress states for plastic deformation or crack initiation may be reached more easily, having strong implications for component's structural integrity.

\section{CONCLUDING REMARKS}

An efficient numerical method for the solution of sliding contact problems is proposed in this paper. Explicit results for the Gauss-Jacobi numerical integration scheme for singular integral equations of the second kind with Cauchy kernels are presented. The resulting discretizations hold valid at chosen collocation points, determined as zeroes of a Jacobi polynomial. As in 
all Gaussian quadratures, the final quadrature formulae involve fixed nodal points and provide exact results for polynomials of degree $2 n-1$, where $n$ is the number of nodes. The method captures correctly the singular or regular behaviour of the traction distribution near the edges of contact.

Two typical example problems for sliding contacts are solved. One is the frictional sliding in the case of a plane Hertzian contact. Comparison between the numerical result and the analytical solution provides a good demonstration of the validity of this method. The sliding contact between a flat punch with rounded corners and a flat surface of a semi-infinite elastic solid is treated numerically. The effect of the friction coefficient on the normal traction distribution is studied. Numerical results show that the increase in the peak normal traction compared to the frictionless case is mild provided the friction coefficient does not exceed about 0.6. For these cases our result confirms that frictionless contact approximation is acceptable. The numerical solutions obtained in this paper have also been extensively validated against finite element simulations, and showed excellent agreement, within the accuracy limits afforded by the finite element models. It is worth noting that the computational time required to obtain the solution using the methods described in the present paper was at least a factor of 20 less than that needed to solve the corresponding finite element model, not counting the data preparation time.

The current method has so far been applied to fully sliding contacts, and allows complete, semi-complete, and incomplete contacts to be considered. From the point of view of computational efficiency this method seems preferable to the triangular traction distribution method.

The method would also allow partial slip contact problems to be considered. This class of problems will be studied separately.

\section{REFERENCES}

1. Dundurs J, Stippes M. Role of elastic constants in certain contact problems. Journal of Applied Mechanics 1970; 37:965-970.

2. Keer LM, Dundurs J, Tsai KC. Problems involving a receding contact between a layer and half-space. Journal of Applied Mechanics 1972; 39:1115-1120.

3. Hills DA, Nowell D, Sackfield A. Mechanics of Elastic Contacts. Butterworth-Heinemann Ltd.: Oxford, 1993.

4. Johnson KL. Contact Mechanics. Cambridge University Press: Cambridge, MA, 1985.

5. Dorogoy A, Banks-Sills L. Shear loaded interface crack under the influence of friction: a finite difference. International Journal for Numerical Methods in Engineering 2004; 59:1749-1780.

6. Boddington PHB, Chen K, Ruiz C. The numerical analysis of dovetail joints. Computers and Structures 1985; 20:731-735.

7. Waterhouse RB. Fretting Fatigue. Applied Science Publishers: New York, 1981.

8. Papanikos P, Meguid SA, Stjepanovic Z. Three dimensional nonlinear finite element analysis of dovetail joints in aeroengine discs. Finite Elements Analysis and Design 1998; 29:173-186.

9. Ambrico JM, Begley MR. Plasticity in fretting contact. Journal of the Mechanics and Physics of Solids 2000; 48:2391-2417.

10. Iyer K. Solutions for contact in pinned connections. International Journal of Solids and Structures 2001; 38:9133-9148.

11. Gladwell GML. Contact Problems in the Classical Theory of Elasticity. Sijtoff and Noordhoff: Maryland, U.S.A., 1980.

12. Ma LF, Korsunsky AM. Effect of friction on edge singularities in slip bands. International Journal of Fracture 2003; 123:L143-L150.

13. Ma LF, Korsunsky AM. A note on the Gauss-Jacobi quadrature formulae for singular integral equations of the second kind. International Journal of Fracture 2004; 126:399-405.

14. Ma LF, Korsunsky AM. Fundamental formulation for frictional contact problems of coated systems. International Journal of Solids and Structures 2004; 41:2837-2854. 
15. Korsunsky AM. On the use of interpolative quadratures for hypersingular integrals in fracture mechanics. Proceedings of the Royal Society of London Series A-Mathematical, Physical and Engineering Sciences 2002; 458:2721-2733.

16. Erdogan F, Gupta GD, Cook TS. Numerical solution of singular integral equation. In Methods of Analysis and Solutions of Crack Problems, Sih GC (ed.). Noordhoff: Groningen, 1973; 368-425.

17. Muskhelishvili NI. Some Problems of Mathematical Theory of Elasticity. Nauka: Moscow, 1949.

18. Stroud AH, Secrest D. Gaussian Quadrature Formulas. Macmillan: New York, 1966.

19. Yakimiw E. Accurate computation of weights in classical Gauss-Christoffel quadrature rules. Journal of Computational Physics 1996; 129:406-430.

20. Ciavarella M, Hills DA, Monno G. The influence of rounded edges on indentation by a flat punch. IMechE Part C-Journal of Mechanical Engineering Science 1998; 212:319-328.

21. Hills DA, Sackfield A. Sliding contact between dissimilar elastic cylinders. Journal of Tribology 1985; 107:463-466. 